\title{
Randomized Controlled Clinical Trial of Blood Glucose Awareness Training (BGAT III) in Switzerland and Germany
}

\author{
Hartmut Schachinger, ${ }^{1,2,8}$ Karin Hegar, ${ }^{2,3}$ Norbert Hermanns, ${ }^{4}$ Madeleine Straumann, ${ }^{5}$ \\ Ulrich Keller, ${ }^{3}$ Gabriele Fehm-Wolfsdorf, ${ }^{6}$ Willi Berger, ${ }^{3}$ and Daniel Cox ${ }^{7}$
}

Accepted for publication: March 30, 2005; Published online: October 13, 2005

\begin{abstract}
Although both diabetes and the efficacy of medical management are international issues, psycho-educational interventions might be culturally bound. Blood Glucose Awareness Training (BGAT) is a psycho-educational program for patients with type 1 diabetes mellitus. It is focused on improving recognition and management of extreme blood glucose levels, and is the best documented American psycho-educational program for this purpose. A randomized controlled clinical trial of BGAT's long-term benefits in a non-American setting has been lacking. One hundred and eleven adults with type 1 diabetes mellitus from Switzerland and Germany participated. After a 6 months baseline assessment, subjects were randomly assigned to receive either 2 months of BGAT $(n=56)$ or a physician-guided self-help control intervention $(n=55)$. BGAT improved recognition of low $(p=0.008)$, high $(p=.03)$, and overall blood glucose $(p=0.001)$, and reduced frequency of severe hypoglycemia $(p=0.04)$, without compromising metabolic control. BGAT reduced both the external locus of control $(p<0.02)$ and fear of hypoglycemia $(p<0.02)$. BGAT was efficacious in reducing adverse clinical events and achieving clinically desirable goals in a European, as well as American setting.
\end{abstract}

KEY WORDS: blood glucose awareness training; severe hypoglycemia; psycho-educational program; clinical trial; hypoglycemia fear.

\section{INTRODUCTION}

The central goal of type 1 diabetes mellitus management is to normalize blood glucose levels while avoiding extreme blood glucose values

\footnotetext{
${ }_{1}^{1}$ Department of Clinical Physiology, FB I-Psychobiology, University of Trier, Germany.

${ }^{2}$ Psychosomatic Medicine, Department of Internal Medicine, University Hospital, Basel, Switzerland.

${ }^{3}$ Endocrinology, Diabetology and Clinical Nutrition, Department of Internal Medicine, University Hospital, Basel, Switzerland.

${ }^{4}$ Diabetes Center, Bad Mergentheim, Germany.

${ }^{5}$ Diabetes Outpatient Clinic, Olten, Switzerland.

${ }^{6}$ Lübeck Institute of Behavioral Medicine, Lübeck, Germany.

${ }^{7}$ Center for Behavioral Medicine Research, Department of Psychiatric Medicine, University of Virginia, Charlottesville, Virginia.

${ }^{8}$ To whom correspondence should be addressed at; e-mail: schaechi@uni-trier.de.
}

(hypoglycemia and hyperglycemia). Although the long-term risks associated with hyperglycemia are generally well recognized by patients and health care professionals, the potential hazards of hypoglycemia require more attention (Muhlhauser et al., 2002). During hypoglycemia, significant negative sequelae can occur, including emotional stress for patients (Gonder-Frederick et al., 1997a) and their significant others (Gonder-Frederick et al., 1997b; Stahl et al., 1998), accidents (Cox et al., 2003), and even death (Sovik and Thordarson, 1999). Symptom recognition and accurate detection of extreme blood glucose values are crucial for management of type 1 diabetes mellitus. Symptoms of hyperglycemia and

\footnotetext{
Abbreviations:, BGAT: Blood Glucose Awareness Training, T0: baseline, T1: 1-6 months after intervention, T2: 7-12 months after intervention
} 
hypoglycemia may be minimal, misinterpreted or neglected.

Blood Glucose Awareness Training (BGAT) is a psycho-educational self-management program designed to assist adults with type 1 diabetes mellitus to prevent extreme blood glucose values, and better recognize and treat hyperglycemia and hypoglycemia when they occur. BGAT is typically delivered to outpatients in a group format, over an 8 weeks period. The focus is on teaching patients to identify their individual symptoms suggestive of the extreme blood glucose fluctuations (internal cues) as well as when and how to anticipate blood glucose extremes based on food, exercise, and insulin regimens (external cues).

Participants are instructed to read the appropriate chapter immediately before the class, discuss the personal relevance of the text during the class, and between classes perform homework exercises evaluating the personal relevance of the chapter's content. There have been three editions of BGAT, an initial six chapter version that emphasized internal cues, an eight chapter version focusing on both internal and external cues (BGAT II; Cox et al., 1991, 2001), and an updated version incorporating newer insulins and in which Chapter 8 addresses long-term maintenance (BGAT III; Kinsley et al., 1999). The efficacy of BGAT has been well documented in the USA (Cox et al., 1988, 1991, 2001; Kinsley et al., 1999), with benefits including not only improved blood glucose estimate accuracy, but a dramatic decrease in automobile accidents as well.

However, a European study evaluating BGAT I (Broers et al., 2002) found it to significantly reduce fluctuations in blood glucose but only marginal benefits in improving detection of hypoglycemia and raising questions regarding treatment generalizability. In addition, cultural differences in the applicability of psycho-educational approaches might lead to varying efficacy in a European setting. Therefore, a randomized controlled prospective study of BGAT in European settings was undertaken.

The following hypotheses were tested: (1) BGAT III would lead to improved blood glucose estimation, (2) a reduction in the frequency of extreme blood glucose levels, (3) decrease in the frequency of severe hypoglycemia; and (4) improved psychological functioning in terms of greater internal locus of control and less fear of hypoglycemia. In addition, the effect of BGAT on relevant psychological variables was examined.

\section{SUBJECTS, MATERIALS AND METHODS}

\section{Subjects and Research Centers}

The research was approved by the Ethics Committee of the Basel University Hospital. Six sites in Switzerland and Germany participated in this prospective multi-center study (see appendix for details). Study information was made available to type 1 diabetes mellitus subjects by conventional mail, oral communications, and posters located in the diabetes specialist's office at each site, regardless of severe hypoglycemia history. However, patients known to the physicians as having recurrent severe hypoglycemia were (often successfully) encouraged to participate. The estimated number of type 1 diabets mellitus subjects being aware of this program is about 400. However, it is impossible to determine the exact number. Subjects who indicated interest were invited to a group information session. Those deciding to participate in the study were individually interviewed, as were their diabetes specialists and/or family physicians (focusing on their diabetes treatment, diabetes complications, and psychiatric comorbidity).

Exclusion criteria were uncontrolled physical (i.e., heart or vascular disease) and mental diseases (i.e., depression, eating disorder, and substance abuse) at the time of claimed interest in the program. Somatic comorbidity was assessed by diabetes specialist or family physicians. Diabetes specialists or family physicians transferred their patients to psychiatrists or the psychosomatic medicine outpatient clinic in case of suspected psychiatric or psychosomatic comorbidity. Comorbidity was considered uncontrolled when newly diagnosed or new treatment had to be established within the last 3 months prior to supposed study entry. Study entrance had to be retarded in six patients because of these criteria. Substance dependency and illicit drug use was assessed by self-report data: any use of cannabis products, benzodiazepines, or barbiturates within the last 2 years, and more than $30 \mathrm{~g}$ ethyl alcohol per day. Interestingly, in this sample of middle-aged type 1 diabetes mellitus subjects there was no case qualifying for these criteria.

It was verified that the subjects were on a 'state of the art' intensified insulin regimen, performed three to five injections and at least three blood glucose measurements per day, had a recent adjustment of insulin dose and dosing schedule (if necessary), and routine determination of 
glycosylated hemoglobin (HbA1c) every 3 months. All patients provided informed consent and were made aware of their right to discontinue participation in the study at any time.

\section{Study Design}

A total of 138 subjects decided to participate in the study. After a 6-month baseline assessment period (T0), subjects were randomly assigned to receive either BGAT or to participate in a physicianguided self-help group (control). Because age and diabetes mellitus duration (Gold et al., 1997) are important confounders favoring the occurence of severe hypoglycemia, subjects were matched to controls within each research center for approximate age and duration of diabetes. At each research center, at least one BGAT group and one control group intervention were offered. The randomization procedure was as follows: within each research center, patients were grouped as pairs of approximately the same age and diabetes duration. Then, a random decision was made as to who of the two patients received BGAT. The other patient received the control group intervention.

Fourteen subjects attended fewer than $50 \%$ of the training sessions (eight in the control group and six in the BGAT group) and were not included in the statistical analyses. The two post-intervention measurement periods were at 1-6 months (T1) and 7-12 months (T2) post-intervention. Data was collected at the end of the 6 months period. Thirteen subjects (six controls) who were noncompliant with these followup examinations were also excluded from analyses. Baseline characteristics of the drop-out patients and the remaining 111 subjects ( $80 \%$ of all randomized subjects) are reported in Table I, they did not differ between participating subjects and drop-out patients.
However, glycosylated hemoglobin of drop-outs was $7.26 \%(0.99)$ indicating worse metabolic control in these subjects (Savage non-parametric two-sample test: $p=0.05)$.

Sixty-one percent of the patients had a cohabiting partner. Patients with partners did not differ from patients without partners in hypoglycemic event rate. However, patients with partners had lower glycosylated hemoglobin, especially those patients without severe hypoglycemia in the previous 2 years. The influence of partnership on diabetes-related psychosocial functions will be the focus of another manuscript currently in preparation.

\section{Interventions}

The German version of BGAT III (Lübeck Institute for Behavioral Medicine, Fleischhauerstr. 26, 23552 Lübeck, Germany) was delivered by a physician-psychologist team to groups of 5-12 subjects in eight weekly sessions. To assure sufficient quality across study sites, one of the two BGAT group leaders was a trained psychologist from the Basel core study team. Each BGAT session lasted for about $2 \mathrm{~h}$. The introductory session (Chapter 1 ) is followed by three sessions focusing on the so-called 'internal cues' (physical symptoms; Chapter 2), disruptions in cognitive and motor performance (Chapter 3), and mood changes (Chapter 4). Patients are taught to use these signals to more accurately recognize when their blood glucose is too high or low. The next three chapters focus on how to use 'exogenous cues': previous insulin injections (Chapter 5), food consumption (Chapter 6), and physical exercise (Chapter 7) to better anticipate when blood glucose is likely to rise or fall. Chapter 8 reviews the subject's personal observations from BGAT and discusses ways to maintain BGAT ben-

Table I. Baseline Characteristics

\begin{tabular}{llll}
\hline \multicolumn{1}{c}{ Variable } & BGAT $(n=56)$ & Control $(n=55)$ & Drop-outs $(n=27)$ \\
\hline Sex (female/male) & $25 / 31$ & $21 / 34$ & $12 / 15$ \\
Age (years) & $45(14.4)$ & $47.9(13.1)$ & $48.1(13.4)$ \\
Diabetes duration (years) & $23.1(12)$ & $22.7(12.2)$ & $22.5(13.9)$ \\
Body mass index $\left(\mathrm{kg} / \mathrm{m}^{2}\right)$ & $24.5(4.5)$ & $23.4(3.5)$ & $24.2(4.1)$ \\
During last 2 years before study & & & \\
$\quad$ Patients with severe hypoglycemia (\%) & 64 & 47 & 50 \\
$\quad$ Patients with hypoglycemic coma (\%) & 28 & 25 & 33 \\
During last 6 months before study & & & \\
$\quad$ Motor vehicle accidents $(n)$ & 2 & 2 & 7 \\
$\quad$ Hospitalization (any cause) $(n)$ & 5 & 6 & 1 \\
$\quad$ Diabetic ketoacidosis $(n)$ & 0 & 1 & \\
\hline
\end{tabular}

Note. Severe hypoglycemia (help of others required), Means and SD is reported. 
efits. Weekly homework and preparatory readings were required.

The self-help control group was guided by one physician. Five to twelve subjects participated in three monthly sessions. Each control group session lasted for about $2 \mathrm{~h}$. The focus of the sessions was: 'current problems related to diabetes,' 'stress and diabetes,' 'anatomy and physiology,' 'physical activity,' 'diabetes in the workplace,' 'relationship conflicts,' and 'previous experiences'; however, participants determined the specific issues and timing. There was no homework assigned to the control group.

\section{Dependent Variables}

All the subjects were instructed to use 2 month diaries developed for this study. Diaries were such that patients could easily insert date and time of measurement, blood glucose estimation, actual blood glucose values, and remarks. Important telephone numbers and mailing addresses (study center, psychologists) were provided on the inside of the cover pages. Patients tested blood glucose at least three times daily. The testing schedule was subject to individual patient-physician communications. However, most of the patients tested blood glucose four times: fasting blood glucose (after awakening), pre-prandial blood glucose values (before supper and lunch), and before bed-time. BGAT did not affect the number of blood glucose measurements. Severe hypoglycemia was defined as any hypoglycemic episode for which the help of others was required (Tattersall, 1999). Severe hypoglycemia was assessed by means of these blood glucose diaries, as well as questionnaires at 6 and 12 months assessments. Diaries and questionnaires gave the same results, with only some exceptions. If necessary, patients, partners, and physicians were interviewed to clarify discrepant reports. It was not possible to routinely verify hypoglycemic events against glucometer results because of logistic difficulties. Sixty-one percent of patients shared their household with a partner. Cohabiting partners were always contacted when severe hypoglycemia was reported as to verify the occurrence of this event. Hand-held computers (Clarke et al., 1995) are known to increase the reliability of blood glucose estimations, but do not guarantee correct report of actual blood glucose values. They were not used in the current study. A period of at least 3 consecutive weeks with complete data pairs of blood glucose estimations and measurements was neces- sary for each individual patient and assessment point for the subject to be included in analyses. Blood glucose accuracy index (Clarke et al., 1987; Cox et al., 1989), detection of low $(<4 \mathrm{mmol} / \mathrm{L})$ and high ( $>10 \mathrm{mmol} / \mathrm{L}$ ) blood glucose levels, as well as low and high blood glucose risk index (Cox et al., 1994b; Kovatchev et al., 1998) were calculated according to the published standards. Blood glucose thresholds for hypoglycemia symptoms were reported by the subjects based on regular self monitoring blood glucose, they represent subjective estimates.

Standardized questionnaires were used to assess diabetes specific locus of control (Kohlmann et al., 1995), and both diabetes specific (Bradley, 1994; The DCCT Research Group, 1988) and general (Dahlbert, 1992) quality of life measures.

The diabetes-specific locus of control questionnaire was used at T0 and T2, only. It measures four distinct scales: internalization, externalization, unpredictability, and chance control. Internal consistencies range between (Cronbach's alpha) 0.79 and 0.83 . Test-retest reliability scores (6 months interval) range between $r=0.72$ and $r=0.78$. This questionnaire is available in German, only (Kohlmann et al., 1995).

The Well-Being Questionnaire from Bradley is a validated 22-item instrument containing no somatic items to minimize potential direct effects of poor metabolic control. It was developed specifically for use with diabetic individuals and has been shown to have good reliability and validity (Bradley, 1994). It produces four subscales that assess depression, anxiety, positive well-being, and perceived energy over the previous 7 days. The Well-Being Questionnaire has been applied successfully in the recent research (Paschalides et al., 2004).

The specific Diabetes Quality-of-Life questionnaire was developed for insulin-treated diabetes patients. It measures the scales: satisfaction, impact and diabetes-related worry. All scales were found to have high internal consistencies between 0.66 and 0.92 and excellent test-retest reliability between $r=0.78$ and $r=0.92$ (The DCCT Research Group, 1988).

A 19-item mood questionnaire was also employed which is available in German, only. It measures 4 scales (fatigue, hopelessness, negative mood, and positive mood), validation studies revealed internal consistencies ranging between 0.83 and 0.94 (Dahlbert, 1992).

The patients also completed the Hypoglycemia Fear Survey (Irvine et al., 1994) 'worry' and 'behavior' subscales. The Hypoglycemia Fear Survey 
worry subscale is based on reactions to severe hypoglycemia episodes (Gold et al., 1997). Thus, the impact of BGAT on fear of hypoglycemia was analyzed in the subsample of patients who had experienced a severe hypoglycemic episode within the 2 years, prior to participation in this study.

Glycosylated hemoglobin (HbA1c; upper limit of normal range: $6.1-6.3 \%$ ) was determined by an immuno-enzymatic method (DCA, 2000); this data was provided by diabetes specialists or family physicians.

\section{Statistical Analysis}

A repeated measures Analysis of Variance (ANOVA) was used to examine the impact of treatment (between subjects factor: BGAT vs. Controls) and time (within subjects factor: Baseline/T0 vs. 1-6 month/T1 vs. 7-12 month/T2 follow-up). The primary statistic of concern was a significant interaction term. Contrasts were constructed $a$ priori for comparison of T1 vs. T0 and T2 vs. T0. Including 'severe hypoglycemia in the previous 2 years' as a covariate in the model did not have a significant effect on the results, so to preserve statistical power, the covariate was not included in the final model.

Mean and standard deviation (SD) are reported in tables and text. All the testings were two-tailed. Statistical calculations were performed using SAS (Version 8, WinNT, SAS Institute, Cary, NC, USA).

\section{RESULTS}

There was a marginal tendency (chi square statistic, $p=0.07$ ) for a greater prevalence of severe hypoglycemia during the 2 years before entry into the study in the BGAT group. The incidence of motor vehicle accidents, hospitalization for any cause, and diabetic ketoacidosis was low in both groups (see Table I). Age, diabetes duration, and body mass index were comparable between groups (see Table I).

The blood glucose estimation and measurement pairs per subject and assessment period (BGATT0: $233 \pm 195$ [ $n=54]$, T1: $221 \pm 124[n=45]$, T2: $203 \pm 129 \quad[n=46] ; \quad$ CNT-T0: $233 \pm 90 \quad[n=50]$, T1: $221 \pm 127[n=46]$, and T2: $203 \pm 123[n=47]$ $($ mean $\pm \mathrm{SD})$ ) were sufficiently numerous and comparable in time and between groups.

BGAT led to a decrease in severe hypoglycemic episodes and increased recognition of low and high blood glucose levels, so as to improve accuracy index and subjective recognition threshold for hypo- glycemia symptoms (see Table II). Extreme blood glucose fluctuations and glycosylated hemoglobin were not influenced by treatment (see Table II). Locus of control became less external, and treated subjects also experienced less unpredictability related to diabetes (see Table III). The behavior-subscale scores of the Hypoglycemia Fear Survey decreased in the BGAT group (indicating less endorsement of behaviors related to hypoglycemia fear), but initial values were higher in this group, so the results might or might not be due to the intervention. There was no impact of BGAT on the Hypoglycemia Fear Survey worry subscale when the entire sample was analyzed (see Table IV). However, when only patients with a history of severe hypoglycemia were analyzed, scores on the worry subscale indicated a reduction in fear for the BGAT group (see Table IV). There was no overall effect of BGAT on either diabetes specific or general quality of life measures.

\section{DISCUSSION}

The present study demonstrates BGAT's efficacy in reducing adverse clinical events (severe hypoglycemia), without compromising metabolic control and achieving clinically desirable goals (improved recognition of low and high blood glucose, reduced external locus of control and reduced fear of hypoglycemia) in European settings.

The results of this study are in accordance with previous findings in USA type 1 diabetes mellitus samples. BGAT has been shown to improve the accuracy of blood glucose estimation and detection of hypoglycemia (Cox et al., 1994a, 2001), improve decisions to treat low blood glucose and not to drive while experiencing low blood glucose levels (Cox et al., 2001), reduce frequency of severe hypoglycemia (Cox et al., 1995, 2001), reduce frequency of ketoacidosis (Cox et al., 2001) and motor cycle accidents (Cox et al., 1994a, 2001), reduction in fear of hypoglycemia and increased quality of life (Cox et al., 2001), and improve the counterregulatory response to hypoglycemia (Kinsley et al., 1999). We now can add that BGAT reduced external locus of control and increased predictability concerning diabetes-specific issues, and that BGAT increased hypoglycemia symptom thresholds.

Hypoglycemia unawareness is a major contributor to the problem of severe hypoglycemia. BGAT has been demonstrated to increase the blood glucose awareness effectively in the USA, but a Dutch report 
Table II. Effects of BGAT on Hypoglycemia Rate, Recognition of BG Levels, and Metabolic Control

\begin{tabular}{|c|c|c|c|c|c|c|}
\hline Variable & T0 & $\mathrm{T} 1$ & $\mathrm{~T} 2$ & $\begin{array}{l}\text { Time } \times \text { Group } \\
\text { interaction; } F, p\end{array}$ & $\begin{array}{l}\text { Contrast T1 vs. } \\
\text { T0 group } \\
\text { effect; } F, p\end{array}$ & $\begin{array}{c}\text { Contrast T2 vs. } \\
\text { T0 group } \\
\text { effect; } F, p\end{array}$ \\
\hline \multicolumn{7}{|c|}{ Negative consequences } \\
\hline \multicolumn{7}{|c|}{ Severe hypoglycemia (episodes/6 months) } \\
\hline $\operatorname{BGAT}(n=56)$ & $1.61(3.49)$ & $0.13(0.33)$ & $0.13(0.33)$ & \multirow{2}{*}{$\begin{array}{l}F(2,218)=3.14 \\
p=0.04\end{array}$} & \multirow{2}{*}{$\begin{array}{l}F(1,109)=1.73 \\
p=0.19\end{array}$} & \multirow{2}{*}{$\begin{array}{l}F(1,109)=4.04 \\
p=0.04\end{array}$} \\
\hline Control $(n=55)$ & $1.76(3.71)$ & $1.07(2.85)$ & $1.78(4.56)$ & & & \\
\hline \multicolumn{7}{|c|}{ Recognition of BG levels } \\
\hline \multicolumn{7}{|c|}{ Percent detection of low blood glucose levels } \\
\hline $\operatorname{BGAT}(n=33)$ & $52.7(21.8)$ & $58.2(24.8)$ & $65.2(25.2)$ & \multirow{2}{*}{$\begin{array}{l}F(2,132)=4.92 \\
P=0.008\end{array}$} & \multirow{2}{*}{$\begin{array}{l}F(1,66)=3.79 \\
p=0.05\end{array}$} & \multirow{2}{*}{$\begin{array}{l}F(1.66)=8.39 \\
p=0.005\end{array}$} \\
\hline Control $(n=35)$ & $53.5(28.0)$ & $45.8(28.7)$ & $48.0(25.5)$ & & & \\
\hline \multicolumn{7}{|c|}{ Percent detection of high blood glucose levels } \\
\hline $\operatorname{BGAT}(n=33)$ & $45.0(23.6)$ & $53.1(25.0)$ & $53.7(26.2)$ & \multirow{2}{*}{$\begin{array}{l}F(2,126)=3.54 \\
p=0.03\end{array}$} & $F(1,63)=5.93$ & \multirow{2}{*}{$\begin{array}{l}F(1,63)=2.62 \\
p=0.11\end{array}$} \\
\hline Control $(n=32)$ & $38.8(24.0)$ & $33.5(24.8)$ & $38.2(23.5)$ & & $p=0.02$ & \\
\hline \multicolumn{7}{|l|}{ Accuracy Index } \\
\hline $\operatorname{BGAT}(n=37)$ & $38.8(17.1)$ & $45.1(21.6)$ & $47.3(21.7)$ & \multirow{2}{*}{$\begin{array}{l}F(2,144)=7.04 \\
p=0.001\end{array}$} & $F(1,72)=5.21$ & \multirow{2}{*}{$\begin{array}{l}F(1,72)=11.37 \\
p=0.001\end{array}$} \\
\hline Control $(n=37)$ & $38.5(17.5)$ & $35.9(18.5)$ & $34.6(19.5)$ & & $p=0.02$ & \\
\hline \multicolumn{7}{|c|}{ Subjective hypoglycemia symptom threshold } \\
\hline BGAT $(n=44)$ & $3.08(0.73)$ & $3.38(0.64)$ & $3.30(0.72)$ & \multirow{2}{*}{$\begin{array}{l}F(2,178)=2.97 \\
p=0.05\end{array}$} & $F(1,89)=5.10$ & \multirow{2}{*}{$\begin{array}{l}F(1,89)=1.45 \\
p=0.23\end{array}$} \\
\hline Control $(n=47)$ & $3.25(0.83)$ & $3.29(0.75)$ & $3.34(0.70)$ & & $p=0.02$ & \\
\hline \multicolumn{7}{|c|}{ Extreme BG fluctuations } \\
\hline \multicolumn{7}{|c|}{ Low blood glucose index } \\
\hline $\operatorname{BGAT}(n=43)$ & $2.99(1.54)$ & $2.48(1.34)$ & $2.61(1.32)$ & \multirow{2}{*}{$\begin{array}{l}F(2,170)=0.52 \\
p=0.60\end{array}$} & $F(1,85)=0.76$ & \multirow{2}{*}{$\begin{array}{l}F(1,85)=0.67 \\
p=0.42\end{array}$} \\
\hline Control $(n=44)$ & $2.62(1.43)$ & $2.33(1.44)$ & $2.49(1.73)$ & & $p=0.39$ & \\
\hline \multicolumn{7}{|c|}{ High blood glucose index } \\
\hline $\operatorname{BGAT}(n=43)$ & $6.53(3.29)$ & $6.64(3.37)$ & $6.29(2.82)$ & \multirow{2}{*}{$\begin{array}{l}F(2,170)=0.77 \\
p=0.46\end{array}$} & $F(1,85)=0.00$ & \multirow{2}{*}{$\begin{array}{l}F(1,85)=1.08 \\
p=0.30\end{array}$} \\
\hline Control $(n=44)$ & $5.85(2.92)$ & $5.95(3.64)$ & $6.17(3.35)$ & & $p=0.99$ & \\
\hline \multicolumn{7}{|c|}{ Glycosylatad hemoglobin (HbA1c) } \\
\hline $\operatorname{BGAT}(n=53)$ & $6.93(0.82)$ & $6.93(1.02)$ & $6.93(0.96)$ & \multirow{2}{*}{$\begin{array}{l}F(2,202)=0.06 \\
p=0.94\end{array}$} & $F(1,101)=0.09$ & \multirow{2}{*}{$\begin{array}{l}F(1.101)=0.03 \\
p=0.85\end{array}$} \\
\hline Control $(n=50)$ & $6.91(0.94)$ & $6.95(0.98)$ & $6.94(0.94)$ & & $p=0.76$ & \\
\hline
\end{tabular}

Note. Severe hypoglycemia (help of others required), BGAT: Blood Glucose Awareness Training, Baseline (T0), 1-6 months (T1), and 7-12 months (T2) follow-up, Means and SD is reported.

(Broers et al., 2002) questioned theeffectiveness of BGAT. Those investigators tested BGAT in group vs. individual settings and found the short-term effects of BGAT to be beneficial, but rather modest,

Table III. Effects of BGAT on Locus of Control

\begin{tabular}{cccl}
\hline Variable & T0 & T2 & $\begin{array}{l}\text { Time } \times \text { group } \\
\text { interaction; } F, p\end{array}$ \\
\hline $\begin{array}{l}\text { Locus of control } \\
\text { Internalization }\end{array}$ & & & \\
BGAT $(n=54)$ & $38.9(6.6)$ & $38.6(7.1)$ & $F(1,101)=0.00$ \\
Control $(n=49)$ & $38.4(6.4)$ & $38.1(6.6)$ & $p=0.96$ \\
Externalization & & & \\
BGAT $(n=54)$ & $22.4(7.8)$ & $20.4(8.0)$ & $F(1,101)=5.43$ \\
Control $(n=49)$ & $19.5(8.4)$ & $19.8(8.6)$ & $p=0.02$ \\
Chance control & & & \\
BGAT $(n=54)$ & $9.2(4.6)$ & $8.8(4.4)$ & $F(1,101)=0.10$ \\
Control $(n=49)$ & $9.5(4.9)$ & $9.4(5.2)$ & $p=0.75$ \\
Unpredictability & & & \\
BGAT $(n=54)$ & $27.9(8.2)$ & $24.1(8.1)$ & $F(1,101)=14.6$ \\
Control $(n=49)$ & $26.5(8.4)$ & $27.2(8.9)$ & $p=0.0002$ \\
\hline
\end{tabular}

Note. BGAT: Blood Glucose Awareness Training; the Locus of Control questionnaire was not applied at T1, Baseline (T0) and 7-12 month (T2) follow-up, Means and SD is reported. suggesting that differences between the American and European samples might affect the reaction to BGAT. However, those investigators did not assess long-term effects and used a briefer version of BGAT (six sessions instead of eight) which might have accounted for the actual difference. Their negative finding may also be explained by a lack of power.

Hypoglycemia is the limiting factor in diabetes insulin therapy (Cryer, 1994). A recent analysis estimated the annual rate of severe hypoglycemic episodes (requiring the help of others) to be 1.5 per patient in a nonselected population of type 1 diabetes mellitus patients, $82 \%$ of whom were on intensified insulin therapy (ter Braak et al., 2000). Our sample had twice this incidence of severe hypoglycemia, possibly because our samples are older, have longer diabetes mellitus duration, are receiving intensified insulin treatment, and were selectively encouraged to participate in the study if known to suffer from recurrent severe hypoglycemia.

Surprisingly, some BGAT effects were observed at $\mathrm{T} 1$, and others (such as the reduction of severe hy- 
Table IV. Effects of BGAT on Fear of Hypoglycemia

\begin{tabular}{|c|c|c|c|c|c|c|}
\hline Variable & T0 & $\mathrm{T} 1$ & $\mathrm{~T} 2$ & $\begin{array}{c}\text { Time } \times \text { Group } \\
\text { Interaction; } F, p\end{array}$ & $\begin{array}{c}\text { Contrast T1 } \\
\text { vs. T0 Group } \\
\text { effect; } F, p\end{array}$ & $\begin{array}{c}\text { Contrast T2 } \\
\text { vs. T0 Group } \\
\text { effect; } F, p\end{array}$ \\
\hline \multicolumn{7}{|c|}{ Hypoglycemia fear survey } \\
\hline \multicolumn{7}{|c|}{ Entire sample } \\
\hline \multicolumn{7}{|l|}{ Worry } \\
\hline $\operatorname{BGAT}(n=53)$ & $16.5(12.2)$ & $15.2(12.1)$ & $13.2(9.9)$ & \multirow{2}{*}{$\begin{array}{l}F(2,198)=1.03 \\
p=0.36\end{array}$} & \multirow{2}{*}{$\begin{array}{l}F(1,99)=0.01 \\
p=0.93\end{array}$} & \multirow{2}{*}{$\begin{array}{l}F(1,99)=1.39 \\
p=0.24\end{array}$} \\
\hline Control $(n=48)$ & $15.7(11.7)$ & $14.6(12.2)$ & $14.7(12.9)$ & & & \\
\hline \multicolumn{7}{|l|}{ Behavior } \\
\hline BGAT $(n=51)$ & $14.1(9)$ & $13.7(8.2)$ & $11.6(6.9)$ & \multirow{2}{*}{$\begin{array}{l}F(2,194)=3.47 \\
p=0.03\end{array}$} & $F(1,97)=0.25$ & \multirow{2}{*}{$\begin{array}{l}F(1,97)=4.85 \\
p=0.03\end{array}$} \\
\hline Control $(n=48)$ & $11.3(6.6)$ & $11.6(6.4)$ & $12.2(8.5)$ & & $p=0.62$ & \\
\hline \multicolumn{7}{|c|}{ Positive history of severe hypoglycemia within last 2 years } \\
\hline \multicolumn{7}{|c|}{ Worry } \\
\hline $\operatorname{BGAT}(n=33)$ & $20.6(12.8)$ & $18.8(12.5)$ & $15.3(9.9)$ & \multirow{2}{*}{$\begin{array}{l}F(2,106)=4.42 \\
p=0.01\end{array}$} & $F(1,53)=2.13$ & \multirow{2}{*}{$\begin{array}{l}F(1,53)=7.48 \\
p=0.01\end{array}$} \\
\hline Control $(n=22)$ & $17.2(12.1)$ & $19.1(14.8)$ & $19.3(12.9)$ & & $p=0.15$ & \\
\hline \multicolumn{7}{|l|}{ Behavior } \\
\hline $\operatorname{BGAT}(n=32)$ & $16.3(9.1)$ & $15.1(8.7)$ & $12.6(7.9)$ & \multirow{2}{*}{$\begin{array}{l}F(2,104)=4.1 \\
p=0.02\end{array}$} & $F(1,52)=1.24$ & \multirow{2}{*}{$\begin{array}{l}F(1,52)=6.46 \\
p=0.01\end{array}$} \\
\hline Control $(n=22)$ & $12.4(8.1)$ & $13.5(7.5)$ & $14(8.7)$ & & $p=0.27$ & \\
\hline
\end{tabular}

Note. BGAT: Blood Glucose Awareness Training, severe hypoglycemia (help of others required), Baseline (T0), 1-6 month (T1) and 7-12 month (T2) follow-up, Means and SD is reported.

poglycemia) only at $\mathrm{T} 2$. Intensified care provided by the physician at study entry, or other unspecific study effects may have been responsible for the low frequency of severe hypoglycemia at $\mathrm{T} 1$ in both the groups. The increase of severe hypoglycemia rate in the control group at $\mathrm{T} 2$ suggests that this benefit disappeared after another 6 months, when only BGAT effects were of significant duration. This is consistent with the previous research which indicated that 12 months follow-up effects of BGAT were present regardless of whether booster sessions were offered or not (Cox et al., 2001). BGAT improved the percent detection of high blood glucose values and increased subjective hypoglycemia symptom thresholds at $\mathrm{T} 1$, only. Statistical significance at T2 may have been missed as a consequence of limited power.

Several limitations of this study have to be considered. It is possible that participants in the BGAT arm of the study received more physician attention (two group leaders) than those in the control group condition (one physician). Furthermore, the treatments were not of equal length and control participants were not asked to complete homework assignments. Additionally, hypoglycemia was not an obligate topic in the control group meetings. The appropriateness of the control group intervention is often subject to discussion in behavioral intervention trials. Ethical considerations do not allow for the implementation of inefficient control treatments. Although blinding of interventions is often advocated, behavioral treatments cannot be delivered in a truly blinded fashion. Crossover studies, where patients serve as their own controls, are frequently complicated by the fact that behavioral treatments, especially cognitive oriented interventions, have long lasting effects without return to baseline. Furthermore, behavioral treatments as well as control interventions may be sensitive to cultural differences. These problems cannot be solved within one trial. Only the homogeneity of different studies from different cultural backgrounds allows for the generalization intended.

\section{ACKNOWLEDGMENTS}

This study was supported by the Swiss National Diabetes Foundation (SDG), Basel Diabetes Foundation (DGRBB), Walter-und Margarethe von Lichtenstein Foundation, Freie Akademische Gesellschaft Basel, Lilly Inc., Switzerland, and Astra-Fonds. We thank Prof. Dr. Wolf Langewitz and Dr. Brigitta Wössmer, who helped with clinical supervision and advice. We also thank the following diabetes nurses, who were actively engaged in motivating and referring patients: Cornelia Müller, Vreni Bättig, Regula Schinz, Marco Pavan (all Basel) and Bettina Blaser (Luzern).

\section{Appendix}

The following centers participated in the study: Basel University Hospital (HS, KH, WB and UK), diabetes outpatient center practice, Olten (MS), diabetes clinic, Bad Mergentheim ( $\mathrm{NH})$, diabetes 
outpatient center practice, Solothurn (Ernst Iff), diabetes outpatient center practice, Aarau (Jürg Lareida), diabetes outpatient center practice, Winterthur (Elisabeth Nützi), diabetes outpatient center practice, Luzern (Frank Ackermann), and Kantonspital Luzern (Christoph Henzen).

\section{REFERENCES}

Bradley, C. (1994). The well-being questionnaire. In Bradley, C. (Ed.), Handbook of Psychology and Diabetes: A Guide to Psychological Measurement in Diabetes Research and Practice, Harwood Academic Press, Chur, pp. 89-109.

Broers, S., le Cessie, S., van Vliet, K. P., Spinhoven, P., van der Ven, N. C., and Radder, J. K. (2002). Blood glucose awareness training in dutch type 1 diabetes patients. Short-term evaluation of individual and group training. Diabet. Med. 19(2):157161.

Clarke, W., Cox, D., Gonder-Frederick, L., Carter, W., and Pohl, S. (1987). Evaluating clinical accuracy of systems for selfmonitoring of blood glucose. Diabetes Care 10: 622-628.

Clarke, W. L., Cox, D. J., Gonder-Frederick, L. A., Julian, D., Schlundt, D., and Polonsky, W. (1995). Reduced awareness of hypoglycemia in adults with IDDM. A prospective study of hypoglycemic frequency and associated symptoms. Diabetes Care 18(4): 517-522.

Cox, D., Carter, W., Gonder-Frederick, L., Clarke, W., and Pohl, S. (1988). Blood glucose discrimination training in insulindependent diabetes mellitus patients. Biofeedback Self-Regul. 13: 201-217.

Cox, D., Gonder-Frederick, L., Julian, D., and Clarke, W. (1994a). Long-term follow-up evaluation of blood glucose awareness training. Diabetes Care 17: 1-5.

Cox, D., Gonder-Frederick, L., Julian, D., Cryer, P., Lee, J., Richards, F., and Clarke, W. (1991). Intensive vs. standard blood glucose awareness training (BGAT) with insulin dependent diabetes: Mechanisms and ancillary effects. Psychosom. Med. 53: 453-462.

Cox, D., Gonder-Frederick, L., Lee, J., Julian, D., Carter, W., and Clarke, W. (1989). Effects and correlates of blood glucose awareness training among patients with IDDM. Diabetes Care 12: $313-318$

Cox, D., Gonder-Frederick, L., Polonsky, W., Schlundt, D., Julian, D., and Clarke, W. (1995). A multi center evaluation of Blood Glucose Awareness Training-II. Diabetes Care 18: 523-528.

Cox, D., Gonder-Frederick, L., Polonsky, W., Schlundt, D., Julian, D., Kovatchev, B., and Clarke, W. (2001). Blood Glucose Awareness Training (BGAT-2): Long term benefits. Diabetes Care 24: 637-642.

Cox, D., Penberthy, J., Zrebiec, J., Weinger, K., Aikens, J., Stetson, B., DeGroot, M., Trief, P., Schaechinger, H., and Hermanns, H. (2003). Diabetes and driving: International survey of frequency and correlates. Diabetes Care 26: 2329 2334.

Cox, D. J., Kovatchev, B. P., Julian, D. M., Gonder-Frederick, L. A., Polonsky, W. H., Schlundt, D. G., and Clarke, W. L. (1994b). Frequency of severe hypoglycemia in insulin-dependent diabetes mellitus can be predicted from self-monitoring blood glucose data. J. Clin. Endocrinol. Metab. 79(6): 1659-1662.

Cryer, P. (1994). Banting lecture. Hypoglycemia: The limiting factor in the management of IDDM. Diabetes 43: 1378-1378.

Dahlbert, C. (1992). Subjective well being of young adults. Theoretical and empirical analyses of structure and stability. Zeitschrift für Differentielle und Diagnostische Psychologie 13: 207-220.

Gold, A. E., Frier, B. M., McLeod, K. M., and Deary, I. J. (1997). A structural equation model for predictors of severe hypoglycemia in patients with insulin-dependent diabetes mellitus. Diabet. Med. 14: 309-315.

Gonder-Frederick, L., Clarke, W., and Cox, D. (1997a). The emotional, social, and behavioral implications of insulin-induced hypoglycemia. Semin. Clin. Neuropsychiatry 2: 57-65.

Gonder-Frederick, L., Cox, D., Kovatchev, B., Julian, D., and Clarke, W. (1997b). The psychosocial impact of severe hypoglycemic episodes on spouses of patients with IDDM. Diabetes Care 20: 1543-1546.

Irvine, A., Cox, D., and Gonder-Frederick, L. (1994). The Fear of Hypoglycaemia Scale. In Bradley, C. (Ed.), Handbook of Psychology and Diabetes. Routledge, London, pp. 133-155.

Kinsley, B., Weinger, K., Bajaj, M., Levy, C., Quigley, M., Simonson, D., Cox, D., and Jacobson, A. (1999). Blood Glucose Awareness Training and epinephrine responses to hypoglycemia during intensive treatment in Type 1 diabetes mellitus. Diabetes Care 22: 1022-1028.

Kohlmann, C., Petrak, F., Schuler, M., Krohne, H., Kuestner, E., and Beyer, J. (1995). Diabetes-specific control beliefs: Are changes dependent on critical life events? In Kohlmann, C. and Kulzer, B. (Eds.), Diabetes And Psychology. Diagnostic Approaches. Huber, Bern, pp. 111-118.

Kovatchev, B. P., Cox, D. J., Gonder-Frederick, L. A., YoungHyman, D., Schlundt, D., and Clarke, W. (1998). Assessment of risk for severe hypoglycemia among adults with IDDM: validation of the low blood glucose index. Diabetes Care 21(11): 1870-1875.

Muhlhauser, I., Sawicki, P., Blank, M., Overmann, H., Richter, B., and Berger, M. (2002). Reliability of causes of death in persons with Type I diabetes. Diabetologia 45: 1490-1497.

Paschalides, C., Wearden, A. J., Dunkerley, R., Bundy, C., Davies, R., and Dickens, C. M. (2004). The associations of anxiety, depression and personal illness representations with glycaemic control and health-related quality of life in patients with type 2 diabetes mellitus. J. Psychosom. Res. 57(6): 557-564.

Sovik, O., and Thordarson, H. (1999). Dead-in-bed syndrome in young diabetic patients. Diabetes Care 22(Suppl. 2): B40B42.

Stahl, W., Berger, W., Schaechinger, H., and Cox, D. (1998). Spouse's worries concerning diabetic partner's possible hypoglycemia. Diabet. Med. 15: 619-620.

Tattersall, R. (1999). Frequency, causes, and treatment of hypoglycemia. In Frier, B., and Fisher, B. (Eds.), Hypoglycemia in Clinical Diabetes, Wiley, Chichester, UK, pp. 55-87.

ter Braak, E., Appelman, A., van de Laak, M., Stolk, R., van Haeften, T., and Erkelens, D. (2000). Related articles, links free full text clinical characteristics of type 1 diabetic patients with and without severe hypoglycemia. Diabetes Care 23: $1467-1471$.

The DCCT Research Group (1988). Reliability and validity of a diabetes quality-of-life measure for the diabetes control and complications trial. Diabetes Care 11: 725-732. 